\title{
Charlson comorbidity index helps predict the risk of mortality for patients with type 2 diabetic nephropathy"
}

\author{
You-qun HUANG ${ }^{\S}$, Rong GOU ${ }^{\S}$, Yong-shu DIAO, Qing-hua YIN, Wen-xing FAN, Ya-ping LIANG, \\ Yi CHEN, Min WU, Li ZANG, Ling LI, Jing ZANG, Lu CHENG, Ping FU, Fang LIU ${ }^{\dagger *}$ \\ (Division of Nephrology, West China Hospital of Sichuan University, Chengdu 610041, China) \\ †E-mail: liufangfh@163.com
}

Received Apr. 10, 2013; Revision accepted July 29, 2013; Crosschecked Dec. 25, 2013

\begin{abstract}
Our intent is to examine the predictive role of Charlson comorbidity index $(\mathrm{CCl})$ on mortality of patients with type 2 diabetic nephropathy (DN). Based on the $\mathrm{CCl}$ score, the severity of comorbidity was categorized into three grades: mild, with $\mathrm{CCl}$ scores of 1-2; moderate, with $\mathrm{CCl}$ scores of 3-4; and severe, with $\mathrm{CCl}$ scores $\geq 5$. Factors influencing mortality and differences between groups stratified by $\mathrm{CCl}$ were determined by logistical regression analysis and one-way analysis of variance (ANOVA). The impact of $\mathrm{CCl}$ on mortality was assessed by the KaplanMeier analysis. A total of 533 patients with type $2 \mathrm{DN}$ were enrolled in this study, all of them had comorbidity (CCl score $>1)$, and $44.7 \%(238 / 533)$ died. The mortality increased with $\mathrm{CCl}$ scores: $21.0 \%(50 / 238)$ patients with $\mathrm{CCl}$ scores of $1-2,56.7 \%(135 / 238)$ patients with CCl scores of $3-4$, and $22.3 \%(53 / 238)$ patients with $\mathrm{CCl}$ scores $\geq 5$. Logistical regression analysis showed that $\mathrm{CCl}$ scores, hemoglobin, and serum albumin were the potential predictors of mortality $(P<0.05)$. One-way ANOVA analysis showed that DN patients with higher $\mathrm{CCl}$ scores had lower levels of hemoglobulin, higher levels of serum creatinine, and higher mortality rates than those with lower $\mathrm{CCl}$ scores. The Kaplan-Meier curves showed that survival time decreased when the $\mathrm{CCl}$ scores and mortality rates went up. In conclusion, $\mathrm{CCl}$ provides a simple, readily applicable, and valid method for classifying comorbidities and predicting the mortality of type $2 \mathrm{DN}$. An increased awareness of the potential comorbidities in type 2 DN patients may provide insights into this complicated disease and improve the outcomes by identifying and treating patients earlier and more effectively.
\end{abstract}

Key words: Diabetic nephropathy (DN), Charlson comorbidity index (CCI), Mortality doi:10.1631/jzus.B1300109 Document code: A CLC number: R543

\section{Introduction}

Diabetes mellitus (DM) has emerged as a worldwide epidemic disease with increasing prevalence, particularly in Asia. According to the epidemiological data from a national survey of Chinese people (Yang et al., 2010), the prevalence of total

\footnotetext{
\$ Corresponding author

${ }^{\S}$ The two authors contributed equally to this work

* Project supported by the Science and Technology Research Projects of Sichuan Province (No. 2011SZ0215), China

(C) Zhejiang University and Springer-Verlag Berlin Heidelberg 2014
}

diabetes and pre-diabetes in China reached 9.7\% and $15.5 \%$, respectively, which indicates a public health issue and an enormous financial burden for the country. Diabetic nephropathy (DN), one of the most common microvascular complications of diabetes, is the leading cause of end-stage renal disease (ESRD) in developed countries accounting for approximately $50 \%$ of all cases of patients requiring dialysis, and is the second leading cause of ESRD in China. DN sufferers tend to be elderly and are likely to have a variety of chronic health conditions, such as cerebrovascular, cardiovascular, peripheral vascular, connective tissue, liver, chronic pulmonary diseases 
and tumors, which may further complicate diagnosis for the primary care clinicians and worsen clinical outcome of the patients.

Comorbidities are coexistent diseases to a disease of interest or an index disease, which may directly affect the prognosis of the disease of interest, or indirectly influence the choice of treatment (de Groot et al., 2003; Hall, 2006; Yancik et al., 2007). The Charlson comorbidity index (CCI) is the most widely used comorbidity index (Charlson et al., 1987; de Groot et al., 2003). It was developed to predict the one-year mortality among 604 patients based on comorbidity data obtained from a hospital chart review in a single US hospital (Charlson et al., 1987). It contains 19 issues including diabetes with diabetic complications, congestive heart failure, peripheral vascular disease, chronic pulmonary disease, mild and severe liver disease, hemiplegia, renal disease, leukaemia, lymphoma, metastatic tumor, and acquired immunodeficiency syndrome (AIDS), each of which was weighted according to their potential influence on mortality. Since then, CCI has been adapted and verified as applicable and valid for predicting the outcome and risk of death from many comorbid diseases (Sarfati et al., 2011; Mnatzaganian et al., 2012). However, a paucity of studies regarding the role of CCI in predicting the outcome of mortality in patients with DN has been conducted. Therefore, we investigated the validity and predictive accuracy of CCI on mortality for a hospital-based group of DN patients in China.

\section{Materials and methods}

The group consisted of 533 patients with DN who were hospitalized from January 2003 through March 2010 in West China Hospital of Sichuan University, Chengdu, China. Inclusion criteria were patients with their first admission due to type $2 \mathrm{DN}$, who were aged 18 and above and had urinary albumin excretion of $30 \mathrm{mg} / \mathrm{d}$ and above. Patients with fever, urinary infection, and acute complications of diabetes, such as diabetic ketoacidosis, diabetic hyperosmosic coma, and malignant hypertension, were not included. The study was approved by the Ethics Committee of West China Hospital of Sichuan University, and written informed consent was obtained from each patient prior to enrollment in the study.

At admission, demographic information was recorded including age, gender, past medical history and duration of DM. Blood and laboratory data including hemoglobulin, haematocrit, serum albumin (SA), urinary protein excretion, serum creatinine, triglyceride, total cholesterol, and $\mathrm{HbA} \mathrm{c} \mathrm{c}$ were collected. The severity of comorbid diseases was recorded and scored according to CCI. Patients were divided into three groups: mild, with CCI scores of 1-2; moderate, with CCI scores of 3-4; and severe, with CCI scores $\geq 5$. CCI was calculated according to the scoring system established by Charlson et al. (1987). Cardio-cerebrovascular disease (CVD) of the comorbidities in CCI was defined as a history of cardiac arrhythmia, peripheral vascular disease, cerebral vasculopathy, ischemic heart disease, or chronic heart failure. All patients were evaluated from January 2003 through March 2010. The mode of death was determined from clinical information leading to death. Survival time in months was calculated from the establishment of the diagnosis of $\mathrm{DN}$ to the death of a patient.

All values are given as mean \pm standard error (SE). Binary logistic regression analysis was used in the study. A one-way analysis of variance (ANOVA) was used to compare group differences stratified by $\mathrm{CCI}$, and otherwise Chi-square tests were applied. Mortalities at different median evaluation follow-up time were estimated by the Kaplan-Meier curves characterized by CCI and SA. All statistical tests were two-tailed and $P<0.05$ was considered significant. Statistical analyses were performed using SPSS Version 13.0 software.

\section{Results}

\subsection{Demographic and clinical characteristics of the patients at the first referral}

Demographic and clinical characteristics of the 533 patients are shown in Table 1 . The mean age was 65 years, with $35 \%(188 / 533)$ of the patients aged 70 years or greater (data not shown). The duration of DM varied from 3 to 19 years [(10.9 \pm 7.9$)$ years]. The mean hemoglobin concentration was $106.3 \mathrm{~g} / \mathrm{L}, 25 \%$ (132/533) were below $90 \mathrm{~g} / \mathrm{L}$, and 4\% (20/533) were less than $60 \mathrm{~g} / \mathrm{L}$ (data not shown). The SA levels ranged from 28 to $41 \mathrm{~g} / \mathrm{L}$, with $50.7 \%(270 / 533)$ of 
the patients being less than $35 \mathrm{~g} / \mathrm{L}$. Renal insufficiency, defined by a serum creatinine level $\geq 178 \mu \mathrm{mol} / \mathrm{L}$, occurred in $38.3 \%(204 / 533)$ of the patients, while the highest value recorded was about $470 \mu \mathrm{mol} / \mathrm{L}$. The features of overall serum lipid profile were not prominent; i.e., few patients presented hyperlipidemia in this study (Table 1). Based on the albuminuria and renal function, nearly $80 \%(413 / 533)$ of the patients were categorized to be at Mogensen stages IV and V. Chronic comorbidities were very common in this group of patients, with the mean CCI of 2.9 and an utmost score of 12 . Of all the patients, $42.0 \%(n=224)$ scored $3-4$, and $14.1 \%(n=75)$ scored $\geq 5$. CVDs were prominent $(26.6 \%, 142 / 533)$ including myocardial infarction, angina and expansion of heart failure. The overall mortality rate was $44.7 \%$ (238/533), and the mortality of patients with CCI scores of $1-2$ was $21.0 \%(50 / 238), 56.7 \%$ (135/238) in $3-4$, and $22.3 \%(53 / 238)$ in $\geq 5$. This result shows that most DN patients in the group were at the latter phase of the Mogensen stage and had comorbidities, particularly CVDs. The higher the CCI scores they had, the higher the mortality.

\subsection{Possible predictive factors for outcome of death}

To evaluate the risk of death, a series of factors were analyzed. On binary regression analysis, hemoglobin, SA, and CCI score were significantly correlated with death, whereas age, sex, Mogensen stage, duration of diabetes, urinary protein excretion, serum creatinine, HbAlc and hyperlipidemia did not (Table 2). Hemoglobin levels less than $100 \mathrm{~g} / \mathrm{L}$ were associated with a 2.532 -fold [ $95 \%$ confidence interval (CI): 0.167-0.936; $P=0.035]$ and an increased risk of death compared with hemoglobin levels greater than $100 \mathrm{~g} / \mathrm{L}$. The odds ratio (OR) of SA less than $35 \mathrm{~g} / \mathrm{L}$ was associated with a 3.205 -fold increased risk of death $(95 \%$ CI: $0.137-0.713 ; P=0.006)$ compared with an SA above $35 \mathrm{~g} / \mathrm{L}$. Those with CCI scored 3-4 (moderate group) doubled the risk of death (OR=1.934; 95\% CI: 1.751-5.618; $P=0.003)$, and those with CCI scored $\geq 5$ (severe group) reached a more than 5 -fold risk $(\mathrm{OR}=5.464$; $95 \%$ CI: 0.178 16.949; $P=0.225$ ), even though it is not statistically significant. These results show that $\mathrm{CCI}$ is a strong parameter to evaluate the risk of death in $\mathrm{DN}$.
Table 1 Demographic and clinical characteristics of patients

\begin{tabular}{lcc}
\hline \multicolumn{1}{c}{ Characteristic } & $n$ & Value $^{*}$ \\
\hline Age (year) & 533 & $65.2 \pm 10.8$ \\
Male/female & & $302 / 231$ \\
Duration of diabetes (year) & 533 & $10.9 \pm 7.9$ \\
Hemoglobin (g/L) & 533 & $106.3 \pm 25.7$ \\
Haematocrit (L/L) & 533 & $0.3 \pm 0.08$ \\
Serum albumin (g/L) & 533 & $34.5 \pm 6.9$ \\
Urinary protein excretion (g/d) & 533 & $3.48 \pm 3.42$ \\
Blood urea nitrogen (mmol/L) & 533 & $12.8 \pm 10.1$ \\
Serum creatinine ( $\mu$ mol/L) & 533 & $246.8 \pm 230.0$ \\
Triglyceride (mmol/L) & 533 & $1.9 \pm 1.5$ \\
Total cholesterol (mmol/L) & 533 & $4.6 \pm 1.5$ \\
Low-density lipoprotein (mmol/L) & 533 & $2.7 \pm 1.2$ \\
High-density lipoprotein (mmol/L) & 533 & $1.4 \pm 0.5$ \\
HbA1c (\%) & 533 & $8.7 \pm 2.6$ \\
Mogensen stage at diagnosis of DN & & \\
$\quad$ III stage (\%) & 120 & 22.5 \\
IV stage (\%) & 209 & 39.2 \\
V stage (\%) & 204 & 38.3 \\
CCI score & 533 & $2.9 \pm 1.7$ \\
CCI 1-2 (\%) & 234 & 43.9 \\
CCI 3-4 (\%) & 224 & 42.0 \\
CCI $\geq 5$ (\%) & 75 & 14.1 \\
Cardio-cerebrovascular disease (\%) & 142 & 26.6 \\
\hline Valys are & & \\
\hline
\end{tabular}

${ }^{*}$ Values are expressed as mean \pm SE, percentage, or number

\subsection{Associations of CCI with factors and outcomes in $\mathrm{DN}$ patients}

To further confirm the impact of CCI as a predictive value, the DN patients were categorized into three subgroups according to their CCI scores: group 1 (CCI score 1-2), group 2 (CCI score 3-4), and group 3 (CCI score $\geq 5$ ). Table 3 shows the associations of CCI with various factors and clinical outcomes in the DN patients. Compared with group 1, patients in group 3 were older and had a longer duration of diabetes, lower levels of HbAlc and SA, and higher levels of serum creatinine and mortality rate. The rates of mortality and levels of serum creatinine in groups 2 and 3 were significantly higher than those in group 1 , whereas hemoglobin and SA were significantly lower. The results indicate that with the decline of the renal function, the patients of DN with higher CCI scores had higher risk of mortality. 
Table 2 Predictive variables for death

\begin{tabular}{|c|c|c|c|c|c|c|}
\hline \multirow{2}{*}{ Variable } & \multirow{2}{*}{$B$} & \multirow{2}{*}{$\mathrm{SE}$} & \multirow{2}{*}{$P$} & \multirow{2}{*}{ OR } & \multicolumn{2}{|c|}{$95 \% \mathrm{CI}$ for $\mathrm{OR}$} \\
\hline & & & & & Lower & Upper \\
\hline \multicolumn{7}{|l|}{ Age } \\
\hline$<65$ years & & & & 1 & & \\
\hline$\geq 65$ years & 0.421 & 0.414 & 0.309 & 1.524 & 0.677 & 3.432 \\
\hline \multicolumn{7}{|l|}{ Sex } \\
\hline Female & & & & 1 & & \\
\hline Male & -0.590 & 0.423 & 0.163 & 0.554 & 0.242 & 1.271 \\
\hline \multicolumn{7}{|l|}{ Mogensen stage } \\
\hline III & & & & 1 & & \\
\hline IV & 0.541 & 0.524 & 0.302 & 1.718 & 0.615 & 4.800 \\
\hline V & -0.364 & 1.758 & 0.836 & 0.695 & 0.022 & 21.813 \\
\hline \multicolumn{7}{|c|}{ Duration of diabetes } \\
\hline$<10$ years & & & & 1 & & \\
\hline$\geq 10$ years & 0.079 & 0.396 & 0.842 & 1.082 & 0.498 & 2.353 \\
\hline \multicolumn{7}{|l|}{ Hemoglobin } \\
\hline$\geq 100 \mathrm{~g} / \mathrm{L}$ & & & & 1 & & \\
\hline$<100 \mathrm{~g} / \mathrm{L}$ & 0.929 & 0.440 & 0.035 & 2.532 & 1.068 & 5.988 \\
\hline $\begin{array}{l}\text { Urinary protein } \\
\text { excretion }(\mathrm{g} / \mathrm{d})\end{array}$ & -0.442 & 0.251 & 0.078 & 0.064 & 0.393 & 1.051 \\
\hline \multicolumn{7}{|l|}{ Serum albumin } \\
\hline$\geq 35 \mathrm{~g} / \mathrm{L}$ & & & & 1 & & \\
\hline$<35 \mathrm{~g} / \mathrm{L}$ & 1.163 & 0.421 & 0.006 & 3.205 & 1.403 & 7.299 \\
\hline \multicolumn{7}{|c|}{ Serum creatinine } \\
\hline$<133 \mu \mathrm{mol} / \mathrm{L}$ & & & & 1 & & \\
\hline $133-176 \mu \mathrm{mol} / \mathrm{L}$ & 1.753 & 1.775 & 0.323 & 4.292 & 0.005 & 5.612 \\
\hline$\geq 176 \mu \mathrm{mol} / \mathrm{L}$ & 1.457 & 1.662 & 0.381 & 5.780 & 0.009 & 6.052 \\
\hline \multicolumn{7}{|c|}{ Charlson comorbidity index } \\
\hline $1-2$ & & & & 1 & & \\
\hline $3-4$ & 1.697 & 0.580 & 0.003 & 1.934 & 1.751 & 5.618 \\
\hline$\geq 5$ & 0.660 & 0.543 & 0.225 & 5.464 & 0.178 & 16.949 \\
\hline HbAlc (\%) & 0.027 & 0.083 & 0.744 & 1.027 & 0.873 & 1.209 \\
\hline Hyperlipidemia & 0.075 & 0.393 & 0.848 & 1.078 & 0.429 & 2.004 \\
\hline
\end{tabular}

$B$ : regression coefficient; SE: standard error; OR: odds ratio; CI: confidence interval

\subsection{Effect of CCI on survival analysis}

To investigate the effect of CCI on survival analysis, the Kaplan-Meier method was performed and the survival rates at years 3 and 5 were determined. For all of the 533 patients, the 3 -year survival rates were $57.3 \%, 44.6 \%$, and $8 \%$ in patients with CCI scores of $1-2,3-4$, and $\geq 5$, respectively, while the 5-year survival rates were $3.4 \%, 1.3 \%$, and $1.3 \%$ in patients with CCI scores of $1-2,3-4$, and $\geq 5$, respectively. Interestingly, the Kaplan-Meier plots di- rectly show that earlier death was associated with higher cumulative mortality rates in patients with higher CCI during the evaluations. As Fig. 1 shows, the group with the lowest CCI had the highest survival rate at any time, which points out that even after the diagnosis of DN, those with CCI scores of 3-4 after 3 years had survival rates lower than $60 \%$. To illustrate the interaction between CCI and SA, the Kaplan-Meier plots were stratified by CCI and SA (Fig. 2). The plot further confirmed that the lower CCI was associated with the higher risk of death, regardless of the SA level (Fig. 2). To explore the interaction between CCI and a late referral, the Kaplan-Meier plots were stratified by CCI and the Mogensen stage (Fig. 3). It was further confirmed that the higher CCI was associated with the higher risk of death, regardless of the Mogensen stage (Fig. 3). To explore the interaction between CCI and anemia, the Kaplan-Meier plots stratified by CCI and hemoglobin further confirmed that the higher the CCI was associated with the higher risk of death, regardless of the hemoglobin level (Fig. 4). The results show an inverse relationship between CCI scores and the 3 - and 5 -year survival rates, cumulative mortality rates, and survival time regardless of SA level, Mogensen stage, and hemoglobin level.

\section{Discussion}

The increasing number of DM is the cause of the progressive increase in the number of patients with DN. Diabetes is now recognized as the most common primary comorbid condition associated with ESRD (Ritz et al., 1999; US Renal Data System, 2002). It has been discovered that those that suffer from comorbid diabetes and the associated nephropathy are more likely to die of fatal CVD than to develop ESRD (National Kidney Foundation, 2002; Debella et al., 2011). Therefore, it is an important issue to identify the possible predictors for predicting the mortality outcome of DN, so as to increase the awareness of earlier and more effective therapy to improve the outcome. Previous studies have investigated the risk factors influencing the outcome of patients with DM, chronic kidney disease (CKD), and DN (Ueda et al., 2003; McFarlane et al., 2006; So et al., 2006; McEwen et al., 2007). There are a number of risk factors 
Table 3 Associations of CCI with factors and outcomes in the DN patients

\begin{tabular}{|c|c|c|c|c|}
\hline Characteristics & Group $1(n=234)$ & Group $2(n=224)$ & Group $3(n=75)$ & $P$ \\
\hline CCI score & $1-2$ & $3-4$ & $\geq 5$ & \\
\hline Male (\%) & 50.0 & 57.6 & $74.7^{\triangle^{*}}$ & 0.001 \\
\hline Age (year) & $63 \pm 15$ & $67 \pm 13^{\wedge}$ & $70 \pm 14^{\triangle}$ & 0.000 \\
\hline \multicolumn{5}{|l|}{ Mogensen stage at diagnosis of DN } \\
\hline III stage (\%) & 31.2 & 12.9 & 20.0 & 0.000 \\
\hline IV stage $(\%)$ & 58.5 & 23.2 & 26.7 & 0.000 \\
\hline V stage $(\%)$ & 10.3 & 63.9 & 53.3 & 0.000 \\
\hline Duration of diabetes (year) & $9.2 \pm 7.6$ & $10.3 \pm 7.8^{\mathbf{\Delta}}$ & $11.6 \pm 8.1$ & 0.001 \\
\hline Hemoglobin $(\mathrm{g} / \mathrm{L})$ & $122.2 \pm 22.0$ & $111.7 \pm 22.0^{\mathbf{\Delta}}$ & $90.9 \pm 22.7^{\triangle^{*}}$ & 0.000 \\
\hline Haematocrit (L/L) & $0.38 \pm 0.06$ & $0.35 \pm 0.07^{\mathbf{\Lambda}}$ & $0.29 \pm 0.08^{\triangle}$ & 0.000 \\
\hline Serum albumin $(g / L)$ & $35.2 \pm 4.8$ & $34.1 \pm 7.0^{\boldsymbol{\Lambda}}$ & $32.2 \pm 6.4^{\triangle}$ & 0.000 \\
\hline Urinary protein excretion $(\mathrm{g} / \mathrm{d})$ & $3.33 \pm 3.16$ & $4.46 \pm 4.40$ & $2.84 \pm 2.17$ & 0.082 \\
\hline Blood urea nitrogen $(\mathrm{mmol} / \mathrm{L})$ & $6.15 \pm 3.2$ & $7.43 \pm 3.9^{\boldsymbol{\Lambda}}$ & $17.15 \pm 12.7^{\triangle}$ & 0.000 \\
\hline Serum creatinine $(\mu \mathrm{mol} / \mathrm{L})$ & $88.4 \pm 43.0$ & $104.6 \pm 47.1^{\boldsymbol{\Lambda}}$ & $317.3 \pm 280.6^{\triangle^{*}}$ & 0.000 \\
\hline Triglyceride $(\mathrm{mmol} / \mathrm{L})$ & $1.66 \pm 1.26$ & $1.51 \pm 1.18$ & $1.55 \pm 1.16$ & 0.321 \\
\hline Total cholesterol (mmol/L) & $4.56 \pm 1.42$ & $4.59 \pm 1.93^{\wedge}$ & $4.34 \pm 2.00^{\triangle}$ & 0.002 \\
\hline Low-density lipoprotein $(\mathrm{mmol} / \mathrm{L})$ & $2.65 \pm 1.20$ & $2.71 \pm 1.49^{\mathbf{\Delta}}$ & $2.49 \pm 1.54^{\triangle}$ & 0.002 \\
\hline High-density lipoprotein $(\mathrm{mmol} / \mathrm{L})$ & $1.26 \pm 0.47$ & $1.33 \pm 0.64$ & $1.37 \pm 0.67$ & 0.321 \\
\hline $\mathrm{HbA1c}(\%)$ & $8.5 \pm 3.70$ & $8.4 \pm 3.30$ & $7.3 \pm 3.13^{\triangle}$ & 0.023 \\
\hline Mortality (\%) & 21.4 & $60.3^{\wedge}$ & $72.0^{\triangle}$ & 0.000 \\
\hline
\end{tabular}

Data are expressed as mean \pm SE or percentage. Compared with group $1,{ }^{\triangle} P<0.05$ and ${ }^{\wedge} P<0.01$; compared with group $2,{ }^{*} P<0.05$

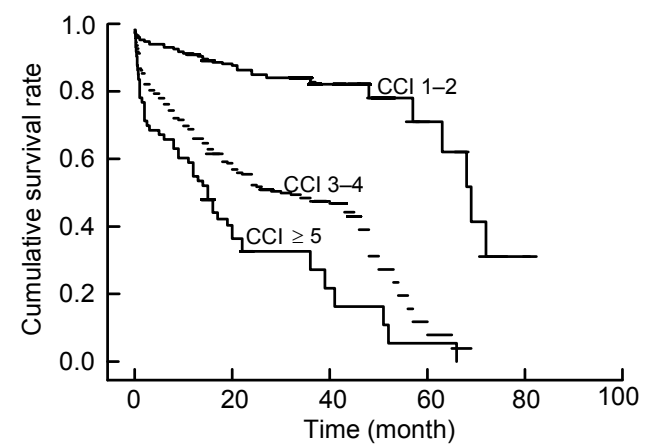

Fig. 1 Kaplan-Meier plot of patients stratified by CCI scores

The months were calculated from the first time diagnosed as DN to death
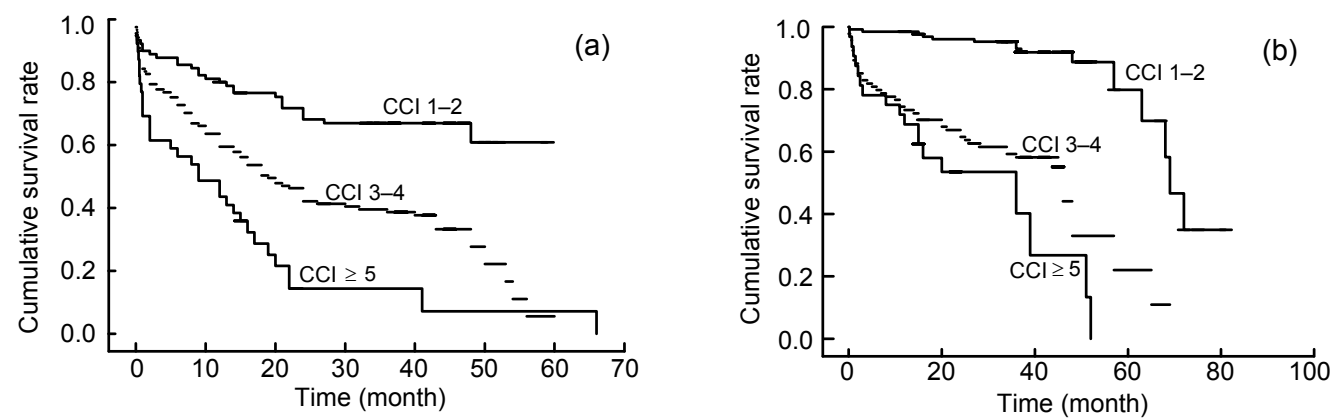

Fig. 2 Kaplan-Meier plots of patients stratified by CCI scores and serum albumin (SA)

(a) $\mathrm{SA}<35 \mathrm{~g} / \mathrm{L}$; (b) $\mathrm{SA} \geq 35 \mathrm{~g} / \mathrm{L}$. The months are calculated from the first time diagnosed as $\mathrm{DN}$ to death 

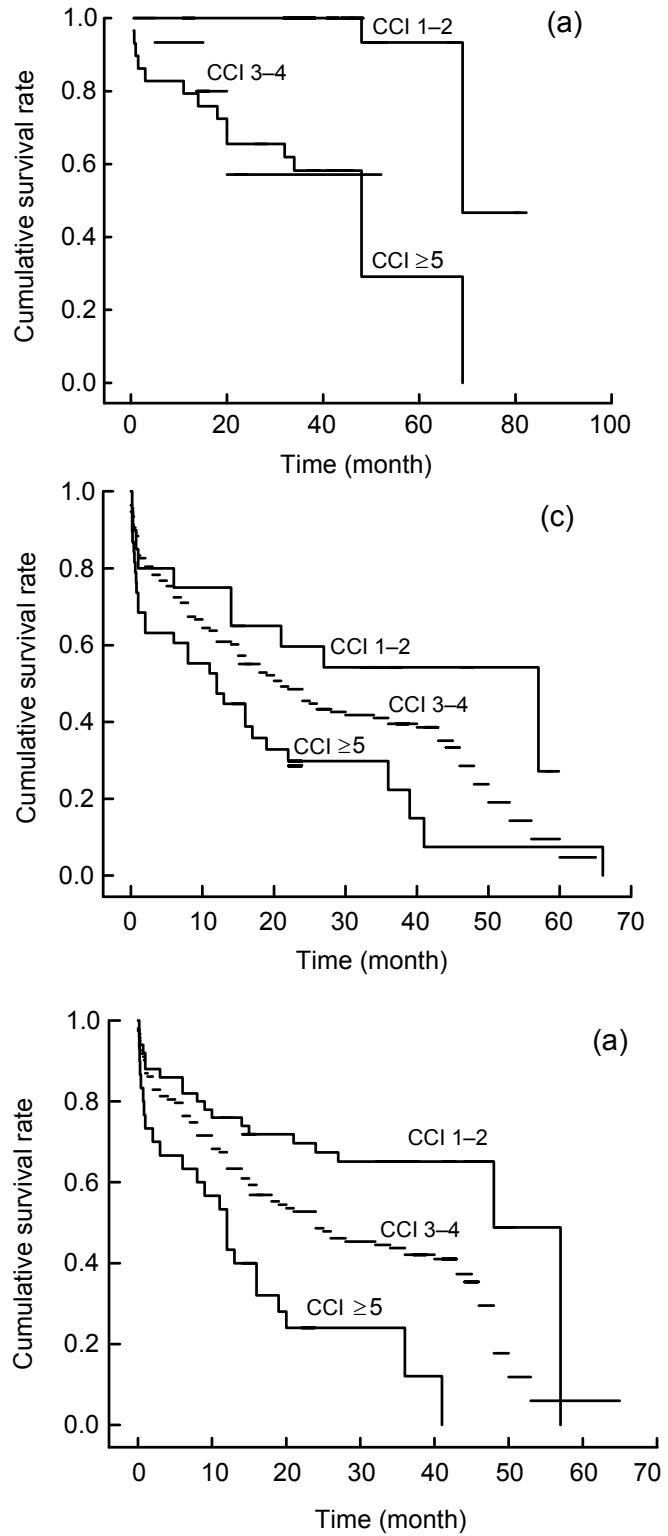

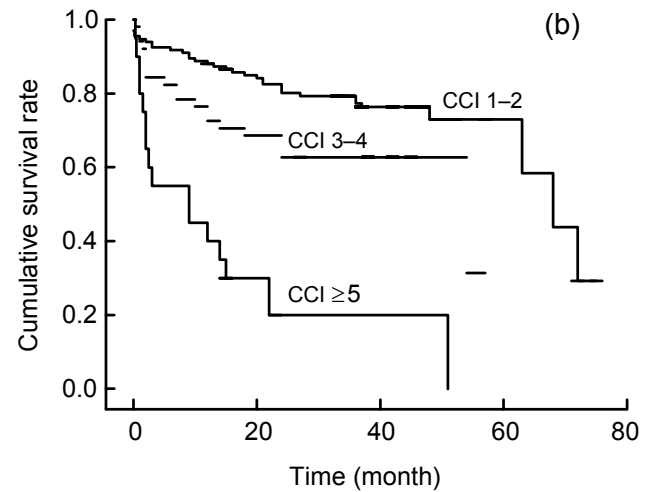

Fig. 3 Kaplan-Meier plots of study patients stratified by CCI scores and Mogensen stages (a) Stage III; (b) Stage IV; (c) Stage V. The months are calculated from the first time diagnosed as DN to death

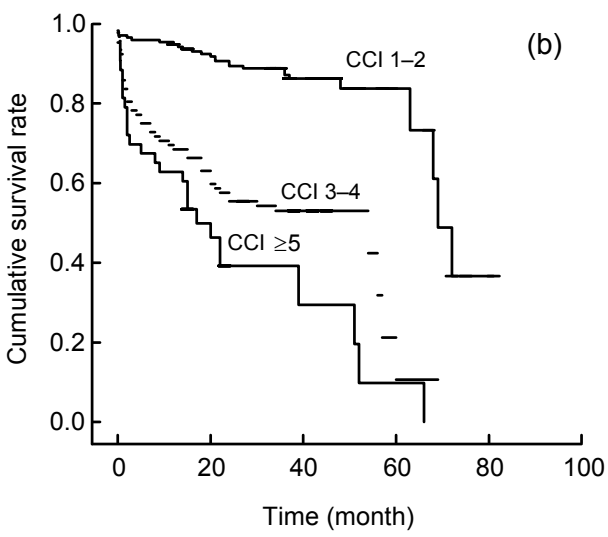

Fig. 4 Kaplan-Meier plots of study patients stratified by CCI and hemoglobin (HGB)

(a) $\mathrm{HGB}<100 \mathrm{~g} / \mathrm{L}$; (b) $\mathrm{HGB} \geq 100 \mathrm{~g} / \mathrm{L}$. The months are calculated from the first time diagnosed as DN to death

associated with the development of DN, some considered "traditional" factors (e.g., old age, male sex, hypertension, diabetes, smoking, high level of low-density lipoprotein (LDL) cholesterol, physical inactivity, and a family history of CVD), and other considered "non-traditional" factors (e.g., albuminuria, anemia, abnormal calcium/phosphate metabolism, electrolytes imbalance, oxidative stress, inflammation, malnutrition, and serum creatinine) (Debella et al., 2011). However, the association of comorbidity and mortality in DN has seldom been addressed. In this paper, we performed a hospital-based study and discovered that the CCI scale helps predict the risk of mortality for patients with type $2 \mathrm{DN}$. In the present study, we found that chronic comorbidities were very common in this group of patients with DN, more than $70 \%$ of the patients had comorbidities, and hemoglobin, SA, and CCI scores were significantly correlated with the risk of death, and CCI significantly increased this risk. 
Comorbidity affects prognosis, therapy, and outcome, and is associated with the decreased health outcomes. According to Feinstein (1970), comorbidity is defined as "any distinct additional entity that has existed or may occur during the clinical course of a patient who has the index disease under study". Another definition is the co-occurrence of multiple diseases in one person (Guralnik, 1996). van den Akker et al. (1998) made a useful distinction between multi-morbidities (i.e., the co-occurrence of multiple chronic or acute diseases and medical conditions in one person) and comorbidity (Feinstein, 1970).

Comorbidity indexes identify the present comorbid diseases and subsequently apply weights or (pathophysiologic) severity ratings for these diseases. $\mathrm{CCI}$ is the most extensively studied comorbidity index for predicting mortality (Charlson et al., 1987; de Groot et al., 2003). Predictive validity of CCI was confirmed by finding many significant relationships of CCI with various criterion outcomes, such as mortality, disability, readmissions, and length of stay (Charlson et al., 1987; Rochon et al., 1996; West et al., 1996; Kieszak et al., 1999; de Groot et al., 2003). Keane et al. (2003) studied factors affecting the prognosis of diabetes in $8733 \mathrm{DM}$ patients and reported that the risk of all-cause mortality doubled when CCI increased with every one point. By using $\mathrm{CCI}$ to predict the mortality outcome of patients with DN, our study investigated an inverse relationship between CCI scores and 3- and 5-year survival rates, cumulative mortality rates, or survival time regardless of SA level, Mogensen stage, and hemoglobin level.

Cardiovascular complications are known as important factors contributing to the increased risk of death in patients with diabetes. CKD is also a powerful risk factor for CVDs. CVDs are an important component in the CCI scoring system. Notably in this study, $26.6 \%$ of all patients suffered from CVDs, and patients with higher CCI had a higher rate of CVDs. The observed prevalence of CVDs is even higher than the incidence of $\mathrm{CV}$ events in the HOPE study (Gerstein et al., 2005). In our group, the cause of death is mainly cardiovascular events (data not shown), including myocardial infarction, congestive heart failure, and cardiac arrests.

Another interesting finding from the current study is that $77.5 \%$ of patients were in the late stage of DN (Mogensen stage IV or V). In accordance with
Lhotta et al. (2003) and Ritz (2005), our findings demonstrate that a late referral to nephrology units was associated with a higher comorbidity score, earlier death, and higher cumulative mortality rates, particularly in patients with DN. Late referral meant that patients had more frequent comorbidities, missed the chance of early reno- and cardio-protective therapy and comorbidities preventive therapy, and did not allow for adequate preparation for renal replacement therapy, which may indicate that the timing of consultation with nephrologists might also be an important determinant of outcome improvement in patients with DN.

Hypoalbuminemia and anemia have been shown to contribute to poor outcomes of patients with CKD. The RENNAL study (Keane et al., 2003) indicated that the risk of developing ESRD decreased by $30 \%$ accompanied per a $5-\mathrm{g} / \mathrm{L}$ increase of SA, and a $10-\mathrm{g} / \mathrm{L}$ fall in the mean SA level was independently associated with the recurrent cardiac failure, ischemic heart disease and cardiac mortality in ESRD patients on hemodialysis (Foley et al., 1996). Hypoalbuminemia (Ueda et al., 2003; So et al., 2006) was found to aggravate the renal function and increase the risk of CVD and directly influence the prognosis of DM. Malnutrition is considered as a strong risk factor for mortality (Suliman et al., 2000). Anemia is a common accompaniment to diabetes, particularly in those with albuminuria or reduced renal function (Thomas et al., 2003), which is also associated with an increased risk of mortality in patients with diabetes. In our group study, we found that patients with higher CCI had lower levels of hemoglobin and SA. In accordance with previous studies, our study clearly illustrated that the levels of hemoglobin and SA were also associated with increased risk of death in patients with DN, while the CCI score showed a stronger correlation with the mortality rate than SA and hemoglobin.

In conclusion, low SA, anemia, CVD, and CCI may serve as predictive factors for the risk of mortality for patients with DN. CCI for classifying comorbid conditions provides a simple, readily applicable and valid method of predicting the risk of mortality for patients with DN. Increased awareness of the potential comorbidities in patients with DN may provide insights into this complicated disease state and possibly improves the efficiency of patient management and outcomes. 


\section{Compliance with ethics guidelines}

You-qun HUANG, Rong GOU, Yong-shu DIAO, Qing-hua YIN, Wen-xing FAN, Ya-ping LIANG, Yi CHEN, Min WU, Li ZANG, Ling LI, Jing ZANG, Lu CHENG, Ping FU, and Fang LIU declare that they have no conflict of interest.

All procedures followed were in accordance with the ethical standards of the responsible committee on human experimentation (institutional and national) and with the Helsinki Declaration of 1975, as revised in 2008 (5). Informed consent was obtained from all patients for being included in the study. Additional informed consent was obtained from all patients for which identifying information is included in this article.

\section{References}

Charlson, M.E., Pompei, P., Ales, K.L., et al., 1987. A new method of classifying prognostic comorbidity in longitudinal studies: development and validation. J. Chronic Dis., 40(5):373-383. [doi:10.1016/0021-9681(87)90171-8]

Debella, Y.T., Giduma, H.D., Light, R.P., et al., 2011. Chronic kidney disease as a coronary disease equivalent-a comparison with diabetes over a decade. Clin. J. Am. Soc. Nephrol., 6(6):1385-1392. [doi:10.2215/CJN.10271110]

de Groot, V., Beckerman, H., Lankhorst, G.J., et al., 2003. How to measure comorbidity: a critical review of available methods. J. Clin. Epidemiol., 56(3):221-229. [doi:10. 1016/S0895-4356(02)00585-1]

Feinstein, A.R., 1970. The pre-therapeutic classification of comorbidity in chronic disease. J. Chronic Dis., 23(7): 455-468. [doi:10.1016/0021-9681(70)90054-8]

Foley, R.N., Parfrey, P.S., Harnett, J.D., et al., 1996. Hypoalbuminemia, cardiac morbidity, and mortality in end-stage renal disease. J. Am. Soc. Nephrol., 7(5): 728-736.

Gerstein, H.C., Pogue, J., Mann, J.F., et al., 2005. The relationship between dysglycaemia and cardiovascular and renal risk in diabetic and non-diabetic participants in the HOPE study: a prospective epidemiological analysis. Diabetologia, 48(9):1749-1755. [doi:10.1007/s00125005-1858-4]

Guralnik, J.M., 1996. Assessing the impact of comorbidity in the older population. Ann. Epidemiol., 6(5):376-380. [doi:10.1016/S1047-2797(96)00060-9]

Hall, S.F., 2006. A user's guide to selecting a comorbidity index for clinical research. J. Clin. Epidemiol., 59(8): 849-855. [doi:10.1016/j.jclinepi.2005.11.013]

Keane, W.F., Brenner, B.M., de Zeeuw, D., et al., 2003. The risk of developing end-stage renal disease in patients with type 2 diabetes and nephropathy: the RENAAL study. Kidney Int., 63(4):1499-1507. [doi:10.1046/j.1523-1755. 2003.00885.x]

Kieszak, S.M., Flanders, W.D., Kosinski, A.S., et al., 1999. A comparison of the Charlson comorbidity index derived from medical record data and administrative billing data. J. Clin. Epidemiol., 52(2):137-142. [doi:10.1016/S08954356(98)00154-1]

Lhotta, K., Zoebl, M., Mayer, G., et al., 2003. Late referral defined by renal function: association with morbidity and mortality. J. Nephrol., 16(6):855-861.

McEwen, L.N., Kim, C., Karter, A.J., et al., 2007. Risk factors for mortality among patients with diabetes: the translating research into action for diabetes (TRIAD) study. Diab. Care, 30(7):1736-1741.

McFarlane, S.I., Salifu, M.O., Makaryus, J., et al., 2006. Anemia and cardiovascular disease in diabetic nephropathy. Curr. Diab. Rep., 6(3):213-218. [doi:10.1007/ s11892-006-0037-4]

Mnatzaganian, G., Ryan, P., Norman, P.E., et al., 2012. Accuracy of hospital morbidity data and the performance of comorbidity scores as predictors of mortality. J. Clin. Epidemiol., 65(1):107-115. [doi:10.1016/j.jclinepi.2011. 03.014]

National Kidney Foundation, 2002. K/DOQI clinical practice guidelines for chronic kidney disease: evaluation, classification, and stratification. Am. J. Kidney Dis., 39(2S): S1-S266.

Ritz, E., 2005. Managing anaemia and diabetes: a future challenge for nephrologists. Nephrol. Dial. Transplant., 20(Suppl. 6):S21-S25.

Ritz, E., Rychlík, I., Locatelli, F., et al., 1999. End-stage renal failure in type 2 diabetes: a medical catastrophe of worldwide dimensions. Am. J. Kidney Dis., 34(5): 795-808

Rochon, P.A., Katz, J.N., Morrow, L.A., et al., 1996. Comorbid illness is associated with survival and length of hospital stay in patients with chronic disability. A prospective comparison of three comorbidity indices. Med. Care, 34(11):1093-1101.

Sarfati, D., Tan, L., Blakely, T., et al., 2011. Comorbidity among patients with colon cancer in New Zealand. N. Z. Med. J., 124(1338):76-88.

So, W.Y., Kong, A.P., Ma, R.C., et al., 2006. Glomerular filtration rate, cardiorenal end points, and all-cause mortality in type 2 diabetic patients. Diab. Care, 29(9): 2046-2052. [doi:10.2337/dc06-0248]

Suliman, M.E., Qureshi, A.R., Bárány, P., et al., 2000. Hyperhomocysteinemia, nutritional status, and cardiovascular disease in hemodialysis patients. Kidney Int., 57(4):1727-1735. [doi:10.1046/j.1523-1755.2000. 00018.x]

Thomas, M.C., MacIsaac, R.J., Tsalamandris, C., et al., 2003. Unrecognized anemia in patients with diabetes: a crosssectional survey. Diab. Care, 26(4):1164-1169. [doi:10. 2337/diacare.26.4.1164]

Ueda, H., Ishimura, E., Shoji, T., et al., 2003. Factors affecting progression of renal failure in patients with type 2 diabetes. Diab. Care, 26(5):1530-1534. [doi:10.2337/ 
diacare.26.5.1530]

US Renal Data System, 2002. USRDS 2002 Annual Data Report: Atlas of End-Stage Renal Disease in the United States. National Institutes of Health, National Institute of Diabetes and Digestive and Kidney Diseases, Bethesda, MD.

van den Akker, M., Buntinx, F., Metsemakers, J.F., et al., 1998. Multimorbidity in general practice: prevalence, incidence, and determinants of co-occurring chronic and recurrent diseases. J. Clin. Epidemiol., 51(5):367-375. [doi:10. 1016/S0895-4356(97)00306-5]
West, D.W., Satariano, W.A., Ragland, D.R., et al., 1996. Comorbidity and breast cancer survival: a comparison between black and white women. Ann. Epidemiol., 6(5): 13-19. [doi:10.1016/S1047-2797(96)00096-8]

Yancik, R., Ershler, W., Satariano, W., et al., 2007. Report of the national institute on aging task force on comorbidity. $J$. Gerontol. A Biol. Sci. Med. Sci., 62(3):275-280.

Yang, W., Lu, J., Weng, J., et al., 2010. Prevalence of diabetes among men and women in China. N. Engl. J. Med., 362(12):1090-1101. [doi:10.1056/NEJMoa0908292]

\section{中文㔍要:}

\section{本文题目：Charlson 合并症指数对 2 型糖尿病肾病死亡率预测作用的研究}

Charlson comorbidity index helps predict the risk of mortality for patients with type 2 diabetic nephropathy

研究目的：探讨 Charlson 合并症指数 (CCI) 对 2 型糖尿病肾病死亡率的预测作用。

研究方法: 建立 2 型糖尿病肾病研究队列, 根据 $\mathrm{CCI}$ 评分将患者合并症的严重程度分为三度: 轻度 (CCI 1-2 分）、中度（CCI 3-4 分）、重度（CCI $\geq 5$ 分）。将影响死亡率的因素及按 CCI 分层后 的组间差异指标进行 Logistic 回归分析及方差分析（ANOVA）。Kaplan-Meier 生存曲线分析 CCI 指数对生存时间及死亡率的影响。

重要结论: 533 例 2 型糖尿病肾病患者纳入研究。所有患者的 CCI 评分均大于 1, 44.7\% (238/533) 的 患者死亡。患者的死亡率随 CCI 评分增加而增加, CCI 1-2 分患者的死亡率为 21.0\%(50/238), CCI 3-4 分患者的死亡率为 56.7\% (135/238)，CCI $\geq 5$ 分患者的死亡率为 $22.3 \% （ 53 / 238 ） 。$ Logistic 回归分析显示 CCI 评分、血红蛋白和血浆白蛋白水平是患者死亡率的预测因子 $(P<0.05)$ 。方差分析结果显示，与 $\mathrm{CCI}$ 评分相对较低的患者相比, CCI 评分越高的患者其 血红蛋白水平较低, 而血清肌酐较高, 死亡率也更高。Kaplan-Meier 生存曲线显示 CCI 评分 越高的患者生存时间越短。CCI 评分是一种简单易行且实用的评估疾病合并症的方法, 可用 于预测 2 型糖尿病肾病患者的死亡率。关注 2 型糖尿病肾病患者的合并症将有利于早期、有 效治疗及改善预后。

关键词组：糖尿病肾病; Charlson 合并症指数; 死亡率 